\title{
Review
}

\section{Remote Patient Monitoring via Non-Invasive Digital Technologies: A Systematic Review}

\author{
Ashok Vegesna, PharmD, ${ }^{1,2}$ Melody Tran, PharmD, ${ }_{1}^{2,3}$ \\ Michele Angelaccio, $B A^{2}$ and Steve Arcona, $P h D^{2}$ \\ ${ }^{1}$ Jefferson College of Population Health, Philadelphia, Pennsylvania. \\ ${ }^{2}$ Novartis Pharmaceuticals Corporation, East Hanover, \\ New Jersey. \\ ${ }^{3}$ Scott \& White Health Plan, Temple, Texas.
}

demonstrating the utility of noninvasive RPM in various patient populations. Future research is needed to determine the effectiveness of RPM via noninvasive digital technologies in delivering patient healthcare benefits and the feasibility of large-scale implementation.

Keywords: e-health, m-health, telehealth, telemedicine

\section{Introduction}

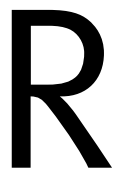
emote patient monitoring (RPM) has enhanced clinicians' ability to monitor and manage patients in nontraditional healthcare settings. RPM uses digital technologies to collect health data from individuals in one location, such as a patient's home, and electronically transmit the information to healthcare providers in a different location for assessment and recommendations. ${ }^{1-3}$ More specifically, noninvasive technologies are now commonly being integrated into disease management strategies to provide additional patient information, with the goal of improving healthcare decision-making. ${ }^{2,4-8}$

Digital technologies are continually being adopted as an additional method for healthcare systems to increase patient contact and augment the practice of preventive medicine. ${ }^{1,2,9}$ Healthcare professionals have the ability to share health data with remotely based clinical experts for consultation, saving time and expense for practitioners and patients, and actively managing treatments for those with chronic conditions. ${ }^{1}$ Health data are typically transmitted to healthcare professionals in facilities such as monitoring centers in primary care settings, hospitals and intensive care units, skilled nursing facilities, and centralized management programs, among others. ${ }^{1,4}$ Diversity exists in the design of noninvasive digital technologies for RPM as well as in the role of the patient. For example, some noninvasive digital devices may be automated to capture and transmit health data without any action from the patient (i.e., biosensor or wearable devices); whereas, other technologies may require the patient to submit their own health data through a secure Web site,

(C) Ashok Vegesna et al., 2017; Published by Mary Ann Liebert, Inc. This Open Access article is distributed under the terms of the Creative Commons License (http://creativecommons.org/licenses/by/4.0), which permits unrestricted use, distribution, and reproduction in any medium, provided the original work is properly credited. 


\section{VEGESNA ET AL.}

smartphone, or personal digital assistant (PDA). ${ }^{1,4}$ Common clinical data captured by these technologies include vital signs, weight, blood pressure, oxygen levels, and heart rate., ${ }^{2,4,10,11}$

Publications to date have used different terminology to capture the essence of RPM (e.g., telehealth, telemedicine, $e-H e a l t h)$ and the overall language used to describe RPM is inconsistent and still being established. ${ }^{1-3,9,12,13}$ Inconsistency in terminology may be attributed to the evolution of this particular group of technologies. As science and technology continue to advance, RPM has transitioned from capturing remote patient data through telephone interviews and videoconferencing to utilizing automated devices (e.g., biosensor devices) and focusing on particular patient populations (e.g., chronic diseases). ${ }^{11}$ A systematic review of the term e-health identified 51 unique definitions for the term with no clear consensus on the meaning. ${ }^{12}$ Another review noted the term telemedicine is used interchangeably with the term e-health (i.e., communication networks used to deliver healthcare services or health information from one geographical location to another). ${ }^{11,13,14}$ Definitions may also be based on the patient's health condition and how RPM technologies gather and send health data back into the health system. ${ }^{4,12}$ In light of these variances, this study focused on a patient-centered definition of RPM: an ambulatory, noninvasive digital technology used to capture patient data in real time and transmit health information for assessment by a health professional or for self-management.

While many RPM interventions have been adopted on a small scale, large-scale implementation continues to be a challenge. To gain clinical credibility, many RPM technologies are tasked with the "burden of evidence" by publishing in the peer-reviewed literature. ${ }^{13,15}$ Due to the relative infancy of this realm of healthcare, we aim to summarize the current level of evidence to date by completing a comprehensive systematic literature review, and applying the same level of rigor required in the evaluation of other healthcare interventions. Hence, the objective of this systematic review was to identify studies reporting the use of patientcentered RPM via noninvasive digital technologies in the past decade and describe the key trends, including patient and clinical characteristics and health-related findings by these technologies.

\section{Materials and Methods}

A search was conducted in EMBASE (2005-2015) and Ovid MEDLINE (January 1, 2005 to September 15, 2015). The search strategy is provided in Supplementary Table S1 (Supplementary Data are available online at www.liebertpub.com/ tmj). An extensive list of search terms was used to identify all appropriate technologies.
The study selection criteria were specified based on the Population, Intervention, Comparator, Outcomes, Timeframe, and Study Design (PICOTS) format. ${ }^{16}$ The patient population included individuals enrolled in a health-related study where the intervention(s) was consistent with our definition of RPM via noninvasive digital technology. We subcategorized interventions based on technology, using the following definitions:

- Any smartphone or PDA device (or associated software/ application/text messaging) that is used to transmit patient data to the physician/researcher.

- Any wearable device worn or placed on a body part to record a particular physiological change (e.g., respiratory rate sensors or blood pressure monitors).

- Any biosensor device for recording data from biological or chemical reactions (e.g., pulse oximeters or spirometers).

- A computerized system where data are entered by the patient over an internet connection.

- Multiple components containing more than one technology category above (e.g., biosensor device and computerized system ${ }^{\mathrm{i}}$ ).

The comparators for the review included either standard care or other technologies used to collect patient health data. Outcomes included any health-related outcomes captured by the RPM technology as well as the associated costs (if reported). Study designs included both randomized controlled trials (RCTs), observational studies, and systematic reviews published from January 1, 2005 to September 15, 2015; protocols, nonsystematic reviews, case studies, commentaries, and letters or editorials were excluded.

Specific exclusion criteria were applied to narrow the focus of selected studies to those reporting patient health data captured via noninvasive RPM digital technologies. The predefined exclusion criteria included the following: interventions with invasive or implantable digital technology (e.g., implantable cardiac defibrillators, blood glucose monitors) or nondigital technology (i.e., landline telephone as the only source of data transmission); no remote monitoring of patient data (e.g., treatment algorithm); no real-time data capture (i.e., participants can only access the device at prescheduled times); and if the data acquired by a device were limited to only patient-reported outcomes, survey responses, or drug performance/adherence.

\section{STUDY SELECTION PROCESS}

All citations identified from the literature search were screened for relevance. The first level of screening involved an assessment of citation abstracts for relevance by a single

${ }^{\mathrm{i}}$ For example: Greene et al. ${ }^{62}$ incorporated data captured from an online social network, accelerometer, and a scale. 


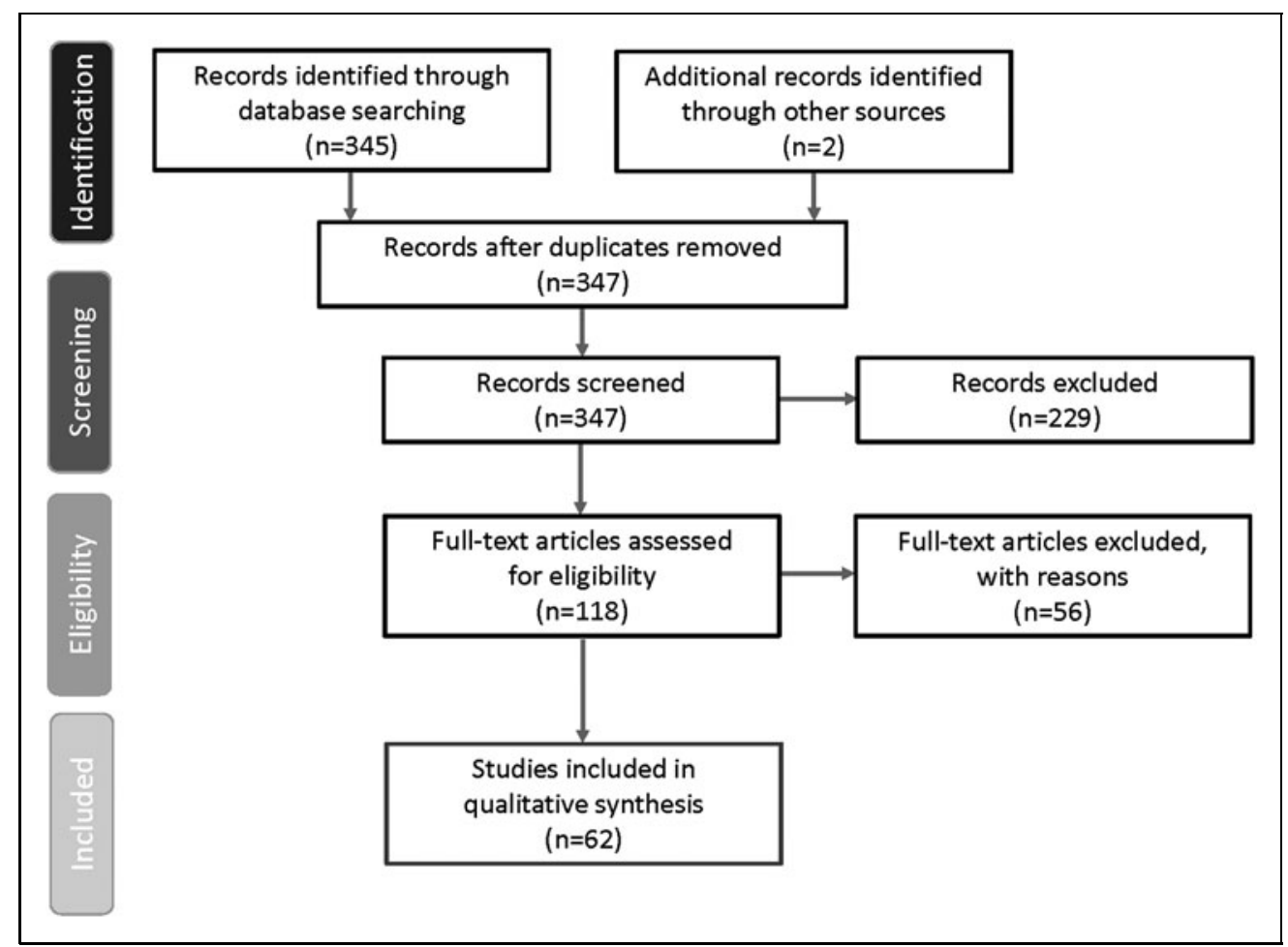

Fig. 1. PRISMA diagram of study selection process. PRISMA, Preferred Reporting Items for Systematic Reviews and Meta-Analyses.

reviewer, based on specific exclusion criteria. Two reviewers were consulted to determine whether any uncertain abstracts were to be included. The second level of screening involved a review of the full-text articles identified from the level one screening to determine if these studies met the predefined inclusion and exclusion criteria listed above. This was undertaken by a single reviewer with four additional reviewers consulted to determine whether any uncertain articles were to be included or excluded based on the study selection criteria.

\section{QUALITY APPRAISAL OF LITERATURE}

The quality appraisal process utilized the Critical Appraisal Skills Programme (CASP) checklists for RCTs, cohort studies, and case-control study designs. ${ }^{17}$ The CASP checklists evaluate the trustworthiness and relevance of studies based on three key areas: study validity (bias), study results (clinical importance and degree of certainty), and relevance (generalizability to patient or population of interest). Quality appraisal was conducted by two independent reviewers for included studies. Instances of disagreement between the two reviewers were identified and evaluated by a third reviewer. Observational, cross-sectional studies are not supported by a CASP quality appraisal checklist. Therefore, the findings from crosssectional studies should be interpreted with caution. ${ }^{18}$

\section{DATA COLLECTION/STUDY VARIABLES}

Due to the high degree of variability in the study designs and objectives, information was extracted to descriptively assess trends and frequencies of the predefined study variables, including the following: technology category, country setting, patient characteristics, and feedback loop. The health outcomes reported by the included studies were broadly defined as positive, negative, or neutral health outcomes, depending on the impact of the intervention on the study outcomes of interest. Clinical or statistical significance was required for a positive categorization as many studies reported only descriptive findings without any supporting statistical analysis. Cost outcomes were also collected, if reported.

\section{Results}

The Preferred Reporting Items for Systematic Reviews and Meta-Analyses (PRISMA) flow diagram (Fig. 1) graphically represents the citations reviewed, included, or excluded during the course of the systematic review process. ${ }^{19}$ The database search identified 345 articles with two additional articles identified from manual searching. Of the 347 articles identified, 62 articles met the study inclusion criteria. Details of each of these studies can be found in Table 1 . 


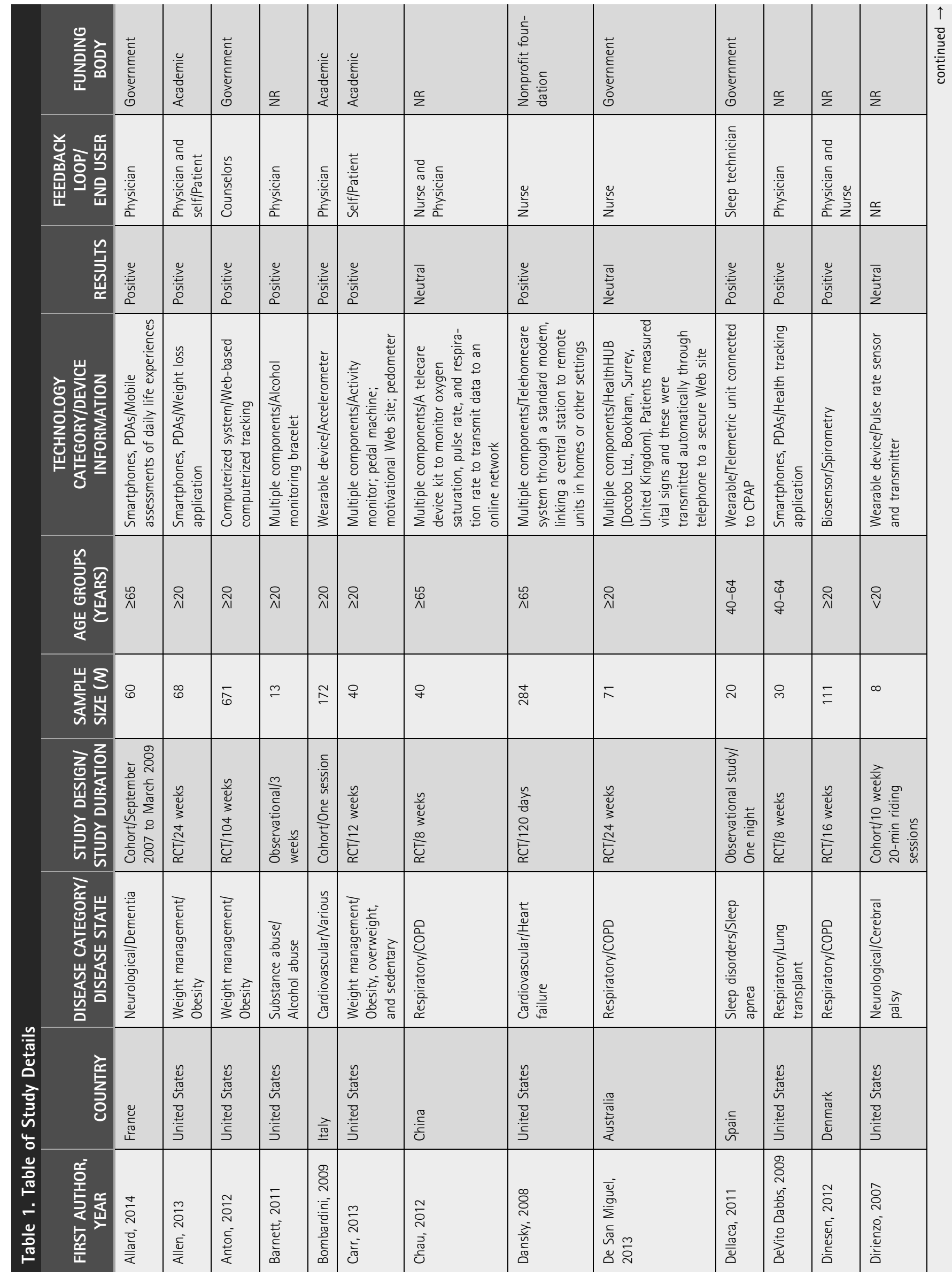




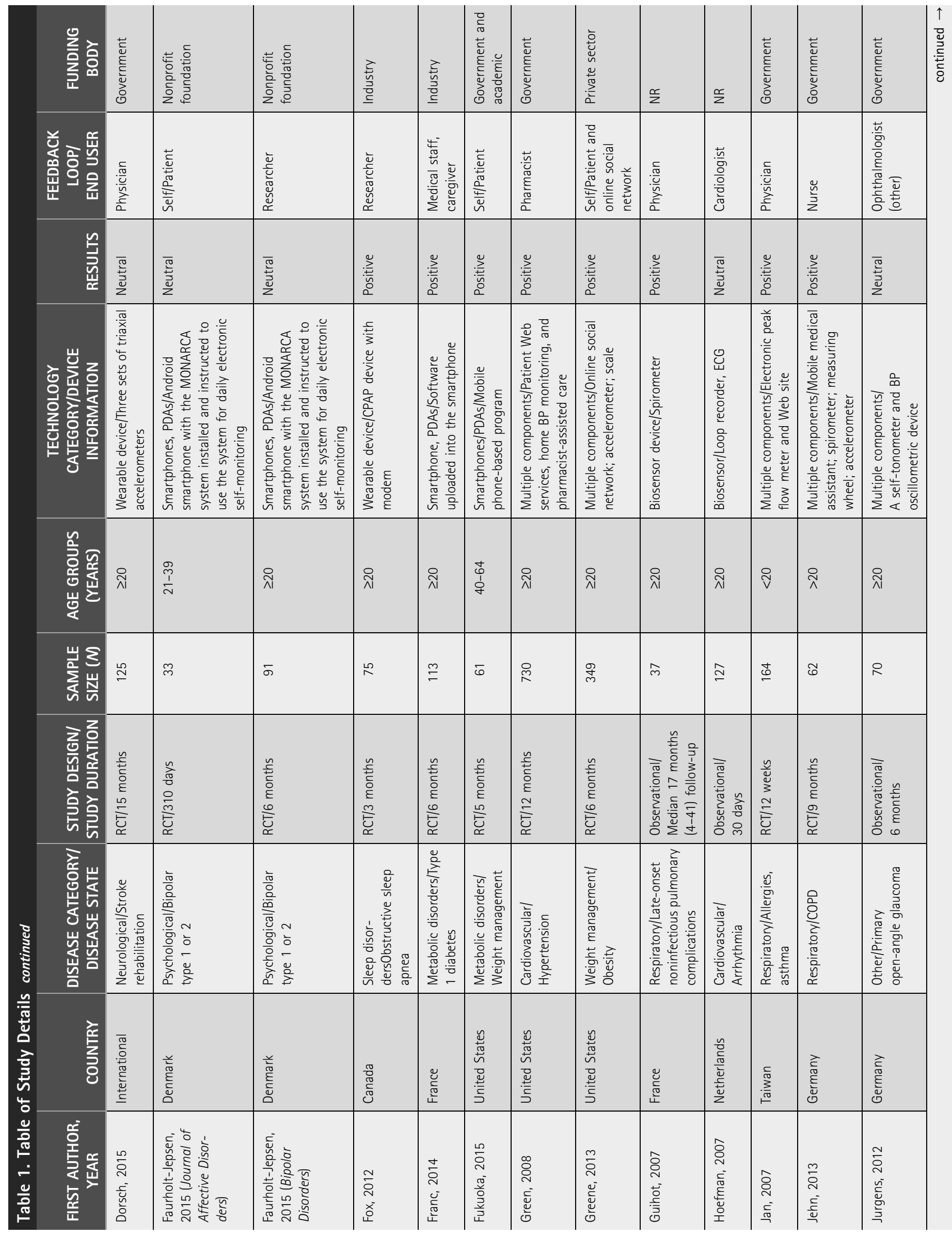




\begin{tabular}{|c|c|c|c|c|c|c|c|c|c|c|c|c|}
\hline 竞言 & & & & & 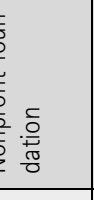 & $\frac{ \pm}{2}$ & 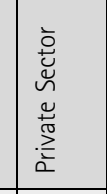 & 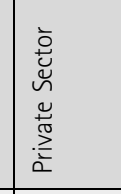 & 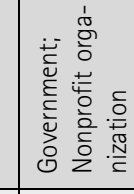 & \pm & $\frac{x}{2}$ & \\
\hline 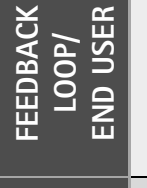 & & & & & 密 & & 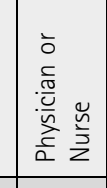 & 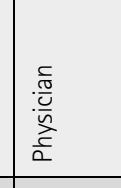 & & 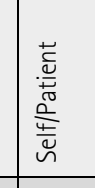 & 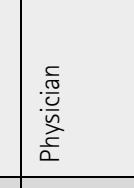 & 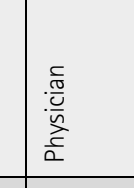 \\
\hline 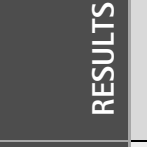 & 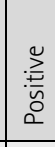 & 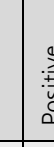 & 美 & & 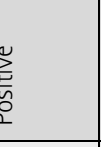 & $\frac{\text { 善 }}{2}$ & $\begin{array}{l}\frac{y}{2} \\
\end{array}$ & $\frac{5}{2}$ & \begin{tabular}{|l}
$\frac{\sqrt{3}}{\underline{\underline{z}}}$ \\
\end{tabular} & 离 & \begin{tabular}{|l}
$\frac{y}{2}$ \\
s.
\end{tabular} & 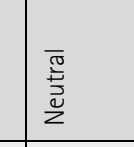 \\
\hline 总 & & & & & 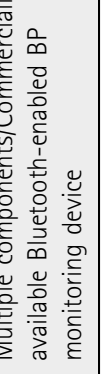 & 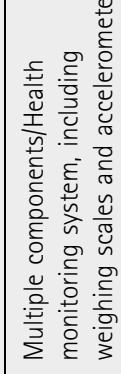 & 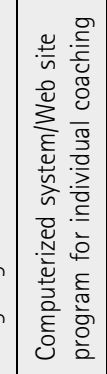 & 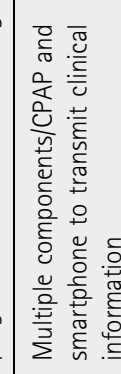 & 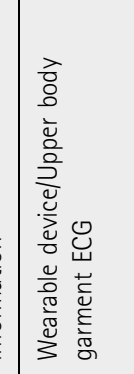 & & 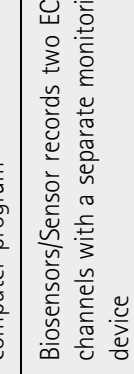 & 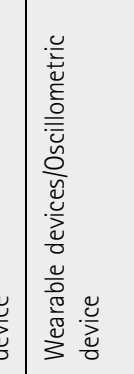 \\
\hline 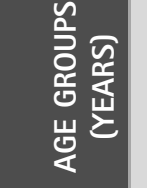 & ฮి & 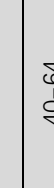 & $\grave{\lambda}$ & & 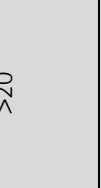 & 旁 & 夁 & ఇิ & $\frac{⿱ 乛}{\frac{q}{\alpha}}$ & 8 & 旁 & 总 \\
\hline 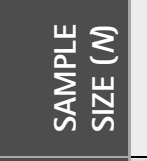 & q & 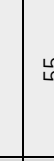 & $=$ & & $=$ & $\therefore$ & $\underline{\dddot{m}}$ & $\cong$ & $\infty$ & $\bar{n}$ & $\Xi$ & $\tilde{g}$ \\
\hline 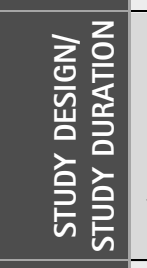 & 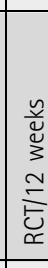 & $\begin{array}{lll}0 \\
0\end{array}$ & 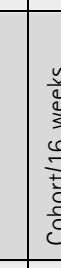 & & 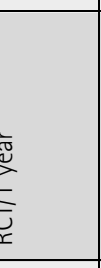 & & 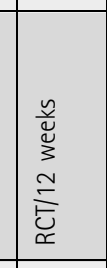 & 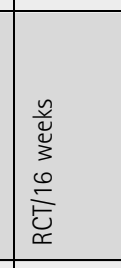 & 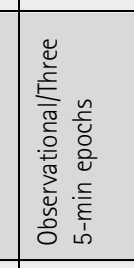 & 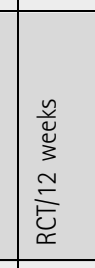 & 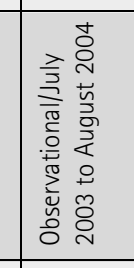 & 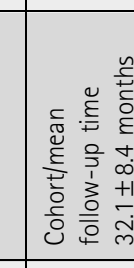 \\
\hline 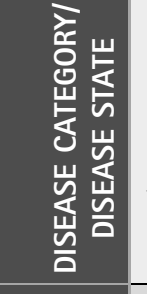 & 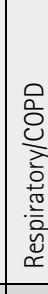 & & 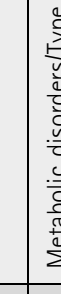 & & 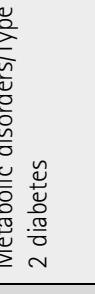 & 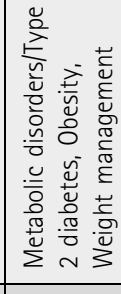 & 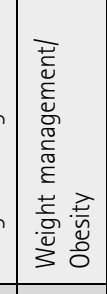 & 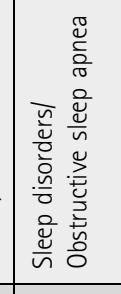 & 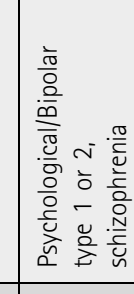 & 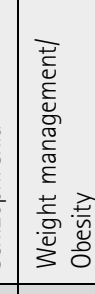 & 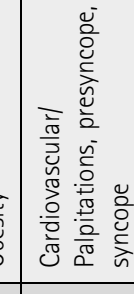 & 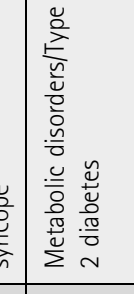 \\
\hline 差 & 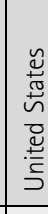 & 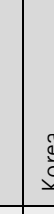 & 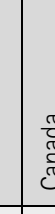 & & 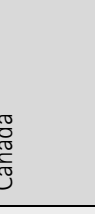 & 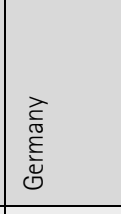 & 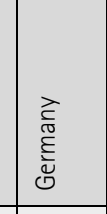 & \begin{tabular}{|l}
$\mid \begin{array}{l}\mathscr{2} \\
\text { 产 }\end{array}$ \\
\end{tabular} & 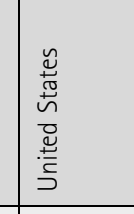 & 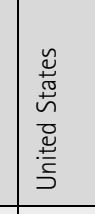 & 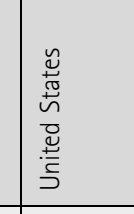 & 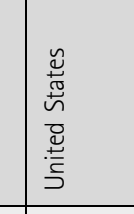 \\
\hline 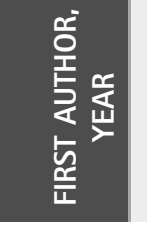 & $\begin{array}{l}\frac{8}{8} \\
\text { : } \\
\frac{1}{2}\end{array}$ & - & & & 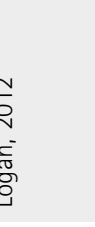 & $\mid$ & $\mid$ & & & 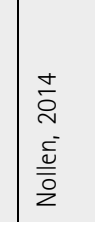 & 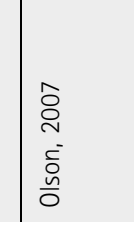 & 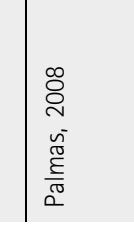 \\
\hline
\end{tabular}




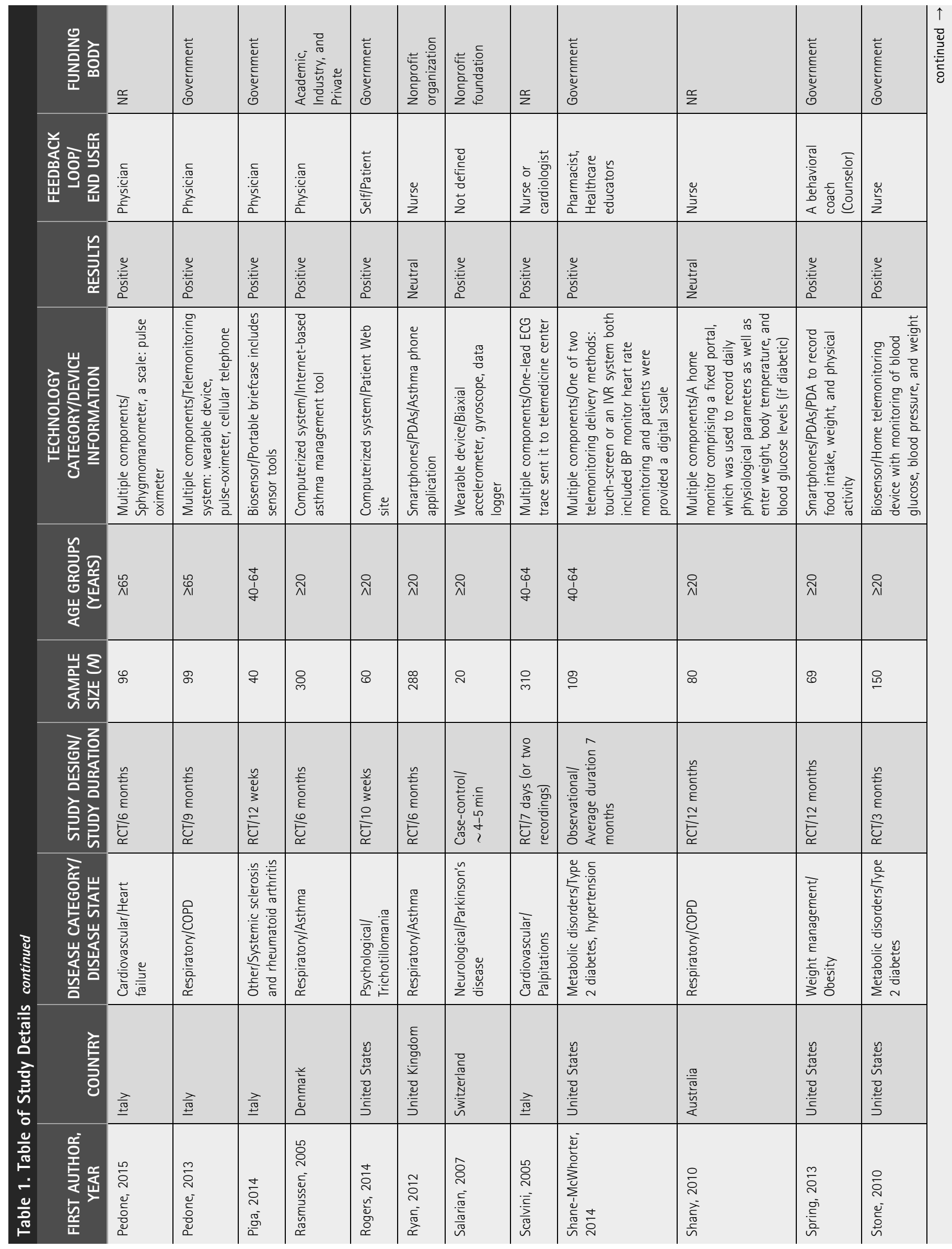




\begin{tabular}{|c|c|c|c|c|c|c|c|c|c|c|c|c|c|}
\hline & 五 & 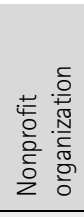 & $\frac{\kappa}{z}$ & 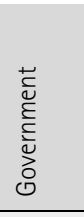 & 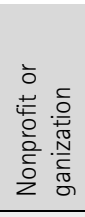 & 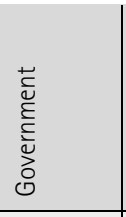 & 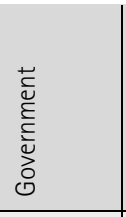 & 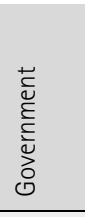 & 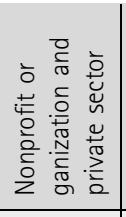 & 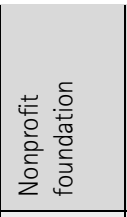 & 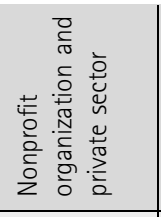 & 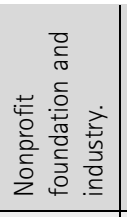 & 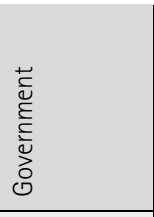 \\
\hline & 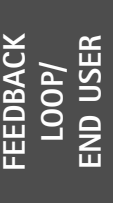 & 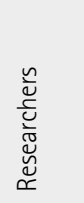 & 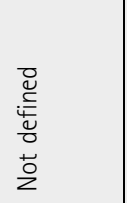 & 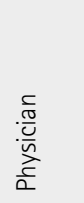 & 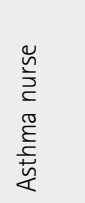 & 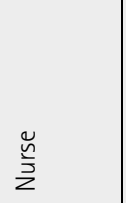 & $\stackrel{\check{n}}{\frac{\tilde{y}}{2}}$ & 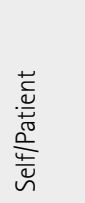 & 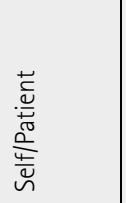 & 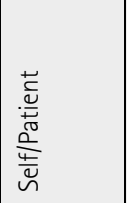 & 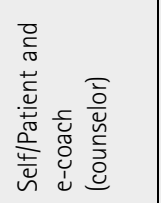 & 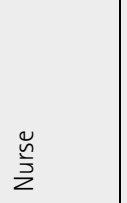 & 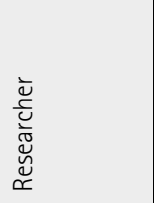 \\
\hline & 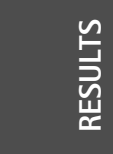 & 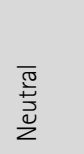 & 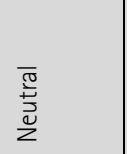 & 竞 & 㝕 & 㝕 & 㝕 & 㝕 & 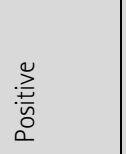 & 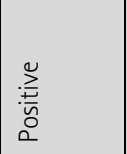 & 竞 & 㝕 & : \\
\hline & 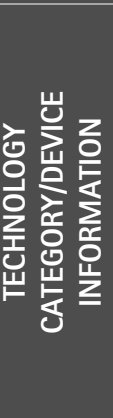 & 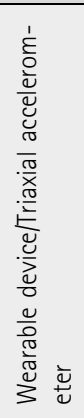 & 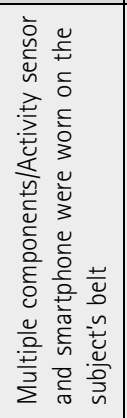 & 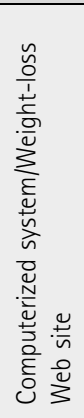 & 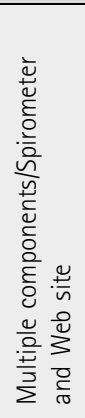 & 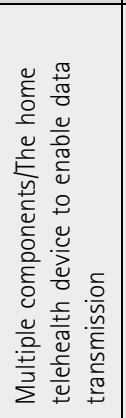 & 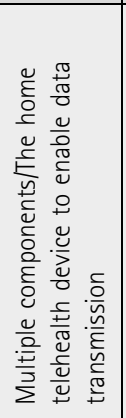 & 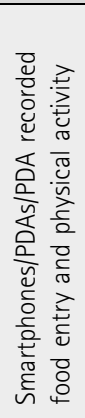 & 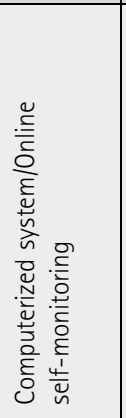 & 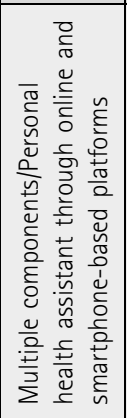 & 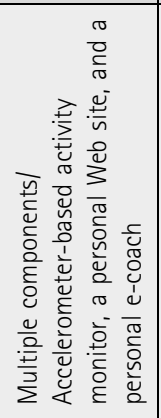 & 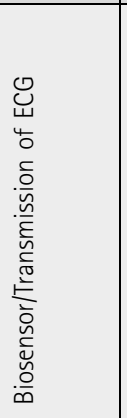 & 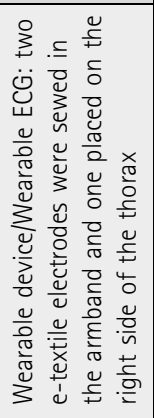 \\
\hline & 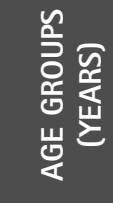 & 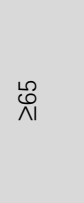 & స્રે & స્તి & స્તે & స્રิ & స્તి & స્તિ & స્તિ & त्रે & & त्रे & $\frac{\infty}{\frac{1}{1}}$ \\
\hline & 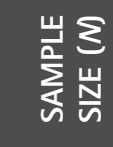 & ๑ & థి & 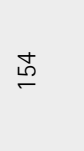 & ষ্ণ & ర్లి & ర్లి & $\stackrel{\circ}{\sim}$ & 오 & $\mathscr{F}$ & $\stackrel{\stackrel{\sim}{\sim}}{\sim}$ & $\stackrel{\text { I }}{\sim}$ & $\stackrel{ }{\circ}$ \\
\hline & 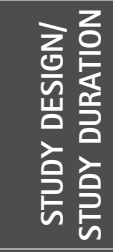 & 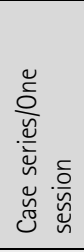 & $\begin{array}{l}n \\
\frac{n}{0} \\
\frac{0}{0} \\
\frac{1}{5} \\
\frac{0}{0} \\
0 \\
\end{array}$ & 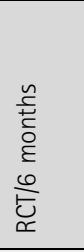 & 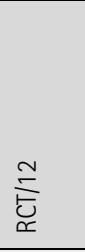 & 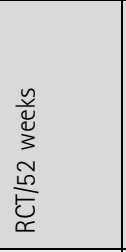 & 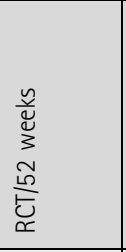 & 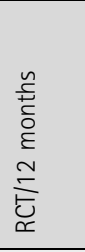 & 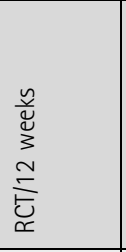 & 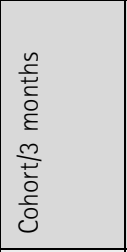 & 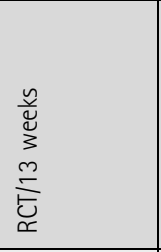 & 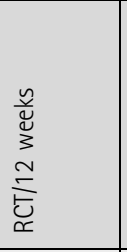 & 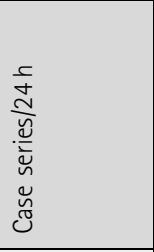 \\
\hline & 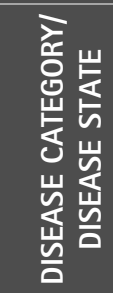 & 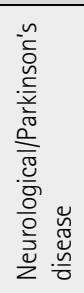 & 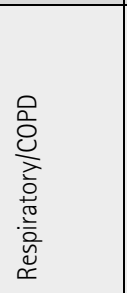 & 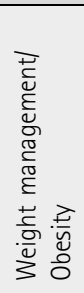 & 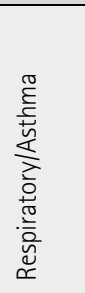 & 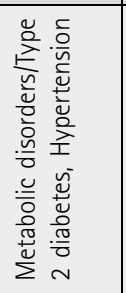 & 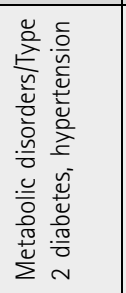 & 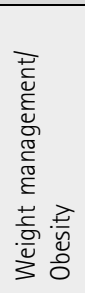 & 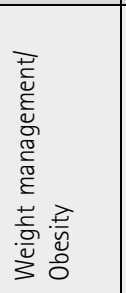 & 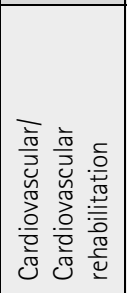 & 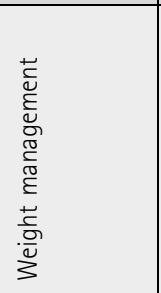 & 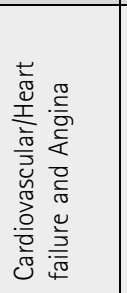 & 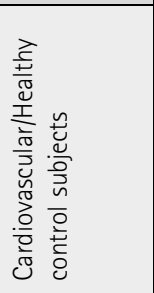 \\
\hline & 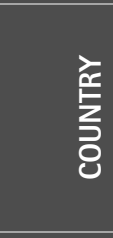 & 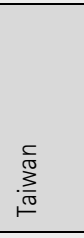 & 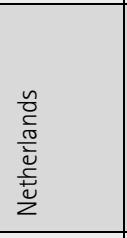 & 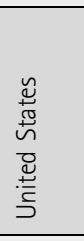 & 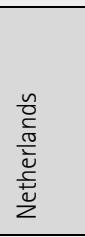 & 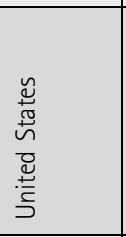 & 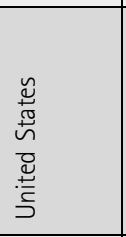 & 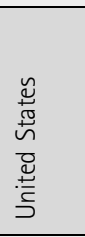 & 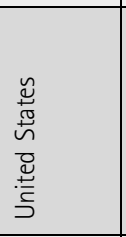 & 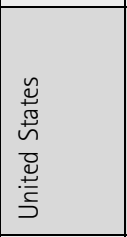 & 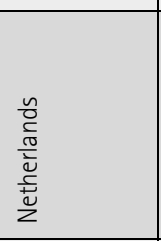 & 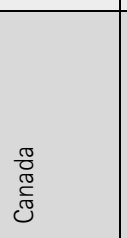 & 胥 \\
\hline 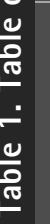 & 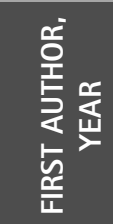 & $\begin{array}{l}\overrightarrow{+} \\
\stackrel{n}{ } \\
\dot{n}\end{array}$ & 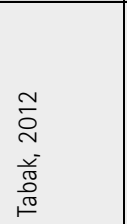 & 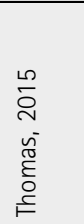 & 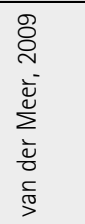 & 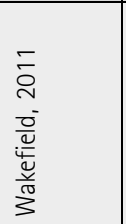 & 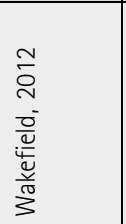 & 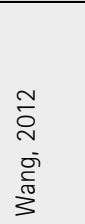 & $\begin{array}{l}\stackrel{m}{0} \\
\dot{\bar{\nu}} \\
\text { oे } \\
\stackrel{0}{3}\end{array}$ & 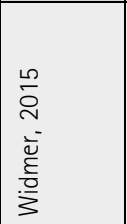 & 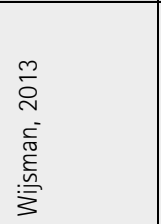 & 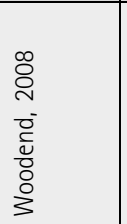 & 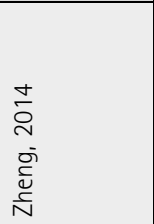 \\
\hline
\end{tabular}




\section{RPM VIA NONINVASIVE DIGITAL TECHNOLOGIES: A SYSTEMATIC REVIEW}

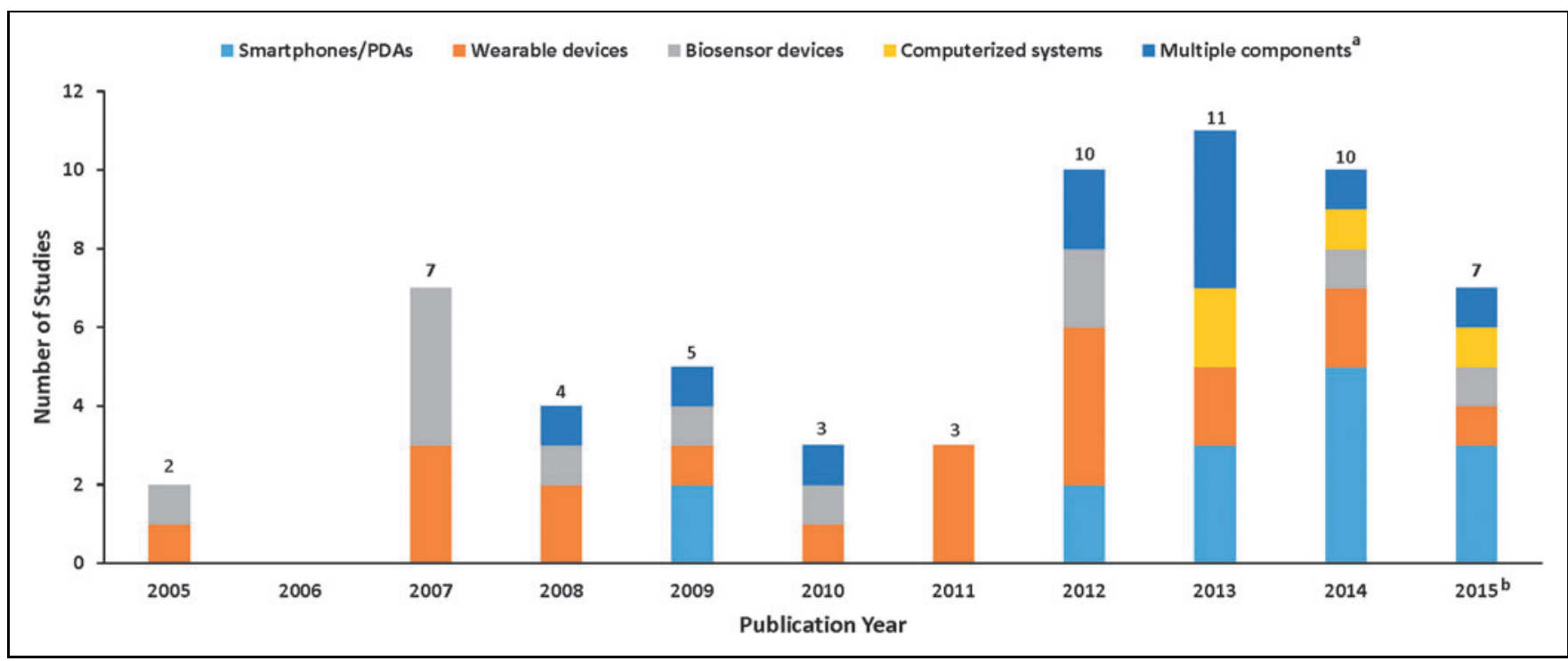

Fig. 2. Identified RPM via Noninvasive Digital Technology Studies (January 1, 2005 to September 15, 2015) ${ }^{\mathrm{a}}$ Multiple components refer to studies containing more than one technology category. ${ }^{b}$ Searches ended September 15, 2015. PDAs, personal digital assistants; RPM, remote patient monitoring.

\section{QUALITY APPRAISAL}

The majority of the 62 studies included in the systematic review used an RCT design. Most of the RCT studies $(N=44)$ received positive responses to the CASP checklist criteria, although one of the criteria regarding blinding of patients and study personnel (to the RPM intervention) was reported by only 10 of the RCTs. The cohort studies $(n=7)$ and casecontrol study $(n=1)$ also had primarily positive responses after being reviewed by the CASP checklists, whereas the remaining studies $(n=10)$ were observational, cross-sectional study designs with no quality appraisal performed.

\section{OVERALL TRENDS: RPM NONINVASIVE DIGITAL TECHNOLOGY}

RPM with noninvasive digital technologies is becoming more available for monitoring and collecting patient healthcare information. Figure 2 illustrates the number of published RPM with noninvasive digital technology studies, by technology category, included in the systematic review over the last decade. Funding of included studies were mainly from government and nonprofit organizations.

The breakdown of technology categories included in the current systematic review is illustrated in Figure 3. Overall, 12 studies were identified using a smartphone or PDA, ${ }^{20-31} 11$ studies utilized wearable devices, ${ }^{32-42} 7$ studies utilized biosensor devices, ${ }^{43-49} 6$ studies included computerized systems, ${ }^{50-55}$ and 26 studies comprised multiple technologies. ii,56-81 $^{-1}$
The patient populations of the included studies were predominantly adults, 20 years of age or older, with only 5\% of studies including children or adolescents ( $<20$ years of age). Many studies recruited specific adult age groups: young adults between 21 and 39 years of age (5\% of studies), older adults between 40 and 64 years of age $(18 \%)$, and seniors $\geq 65$ years old $(10 \%)$. Overall, the majority $(61 \%)$ of studies included a mix of younger and older adult populations. The clinical characteristics of the included studies showed a dominance of chronic disease populations. The most prevalent conditions included the following: 23\% respiratory diseases (e.g., asthma), 18\% weight management, 18\% metabolic diseases (e.g., type 1 and 2 diabetes), and 16\% cardiovascular diseases (e.g., heart failure.)

All studies included elements of a feedback loop, however, the end user or recipient of the captured data varied greatly across the included studies. The majority included physicians or nurses as the main recipient of patient health data $(31 \%$ and $16 \%$, respectively). In $18 \%$ of studies, the end user of the captured data was the patient. These studies primarily utilized smartphones/ PDAs or computerized systems to enable self-monitoring of patient lifestyle behaviors (i.e., caloric intake and exercise).

\section{KEY TRENDS BY NONINVASIVE DIGITAL TECHNOLOGY}

The key trends for each of the technology categories are listed in Table 2, diversity among the technologies was shown across all of the study variables. Only studies on computerized systems were found to be primarily conducted within the

\footnotetext{
${ }^{i i}$ For example: Greene et al. ${ }^{62}$ incorporated data captured from an online social network, accelerometer and a scale.
} 


\section{VEGESNA ET AL.}

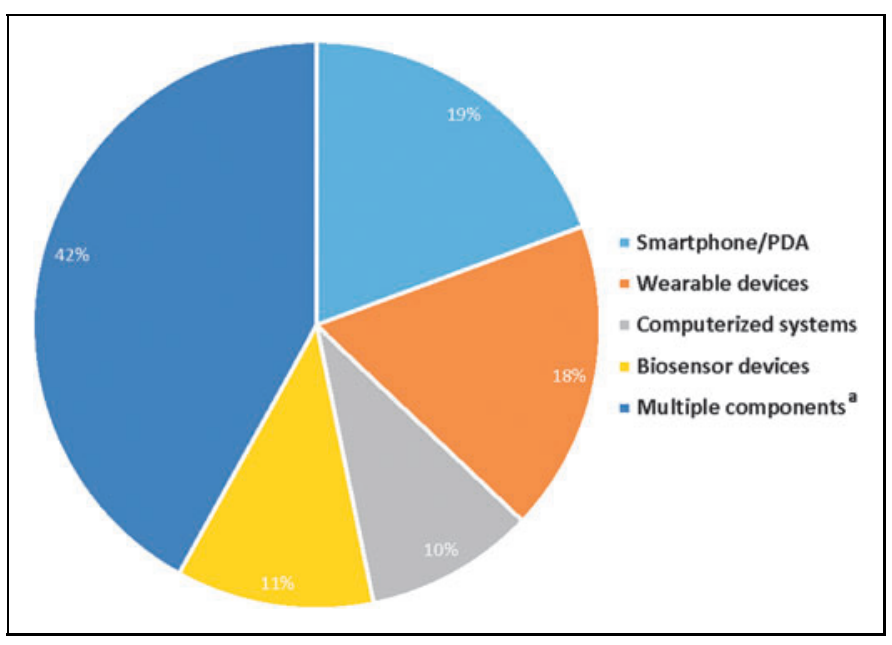

Fig. 3. RPM through Noninvasive Digital Technologies ${ }^{\mathrm{a}}$ Multiple components refer to studies containing more than one technology category.

United States, and studies on smartphones/PDAs were evenly split between U.S. and non-U.S. settings. In terms of clinical and patient characteristics, patients with respiratory disorders/ diseases and metabolic conditions were primarily monitored through multicomponent interventions. In contrast, weight management was mainly monitored through computerized systems, and patients with cardiovascular diseases were mainly monitored using biosensor devices.

The vast majority of studies including adults $\geq 20$ years (incorporating both younger and older adult populations), primarily utilizing multiple-component technologies. Of the three studies including child/adolescent populations, multicomponent interventions, smartphones/PDAs, or wearable devices were used. Similarly, the three studies that specifically recruited young adults (20-39 years) employed smartphones/PDAs or wearable devices. In terms of the feedback loop, smartphones/PDAs and computerized systems most often used the patient as the primary recipient of health data. Wearable devices most often transmitted data to study researchers; whereas biosensor devices and multicomponent interventions predominantly transmitted data to physicians or nurses. Although most of the studies included were RCT designs, the health outcomes reported were diverse and mainly descriptive in nature; therefore, outcomes were classified as primarily positive, negative, or neutral to understand the overall trends. Overall, most studies reported positive health outcomes, ${ }^{\mathrm{iii}}$ including the computerized technologies (100\%), followed by biosensor devices (86\%), multicomponent interventions (81\%), and smartphones/PDAs (58\%). Only studies on wearables devices found an even split between positive and neutral outcomes. ${ }^{\text {iv }}$ Of the six studies that reported cost associated with utilizing an RPM technology, these were also neutral or positive compared with the control group (data not shown). ${ }^{29,43,60,66,69,81}$

\section{Discussion}

RPM is an evolving and growing area of healthcare innovation in terms of research potential and improvement in healthcare services and delivery. ${ }^{5-8,82}$ In the past, telemedicine and e-health were common terms used more broadly to define patient data captured from landlines or videoconferencing interventions. Over the last 10 years, RPM via noninvasive digital technologies has rapidly become a more common way to obtain patient health data. Our systematic literature review is unique in that we identified studies utilizing RPM via noninvasive technologies to investigate the application of these technologies among diverse patient populations and various health conditions.

The majority of studies included in this systematic review were RCTs of multicomponent technologies in adult populations. Chronic conditions, including respiratory diseases, weight management, metabolic diseases, and cardiovascular diseases, were the focus of noninvasive digital RPM interventions, which indicates the high burden of chronic disease to the health system. Healthcare settings where a physician or nurse collected and provided feedback to the patient regarding the remotely captured data represented the majority of studies; the data feedback loop is a critical component in ensuring that RPM interventions have a high impact on patient health. Due to the descriptive nature of many of the included studies, health outcomes were categorized as positive, negative, or neutral (rather than individual measures of efficacy or effectiveness) to allow for an assessment of the overall trends in improving outcomes across all the technologies. The health outcomes data reported were classified as mostly positive across all of the included studies. Key outcomes included self-management (i.e., taking blood pressure or tracking weight) shown to help symptoms severity and reduce patient visits and/or hospitalizations, ${ }^{59,67,79}$ as well as quality of life improvement through activation of self-care behaviors. ${ }^{22,66}$

A limited number of studies have reported healthcare data on the use of RPM via noninvasive digital technologies in the

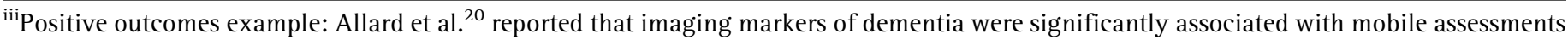
of semantic memory performance.

${ }^{\text {iv }}$ Neutral outcomes example: De San Miguel et al. ${ }^{60}$ reported the telehealth group had nonsignificant reductions in emergency department visits, hospital admissions, and length of stay in comparison with the control group.
} 
Table 2. Key Trends by Noninvasive Digital Technology (Number of Studies)

\begin{tabular}{|c|c|c|c|c|c|}
\hline & $\begin{array}{c}\text { SMARTPHONES/ } \\
\text { PDAS }\end{array}$ & $\begin{array}{l}\text { WEARABLE } \\
\text { DEVICES }\end{array}$ & $\begin{array}{l}\text { BIOSENSOR } \\
\text { DEVICES }\end{array}$ & $\begin{array}{l}\text { COMPUTERIZED } \\
\text { SYSTEM }\end{array}$ & $\begin{array}{c}\text { MULTIPLE } \\
\text { COMPONENTS }\end{array}$ \\
\hline Number of studies, $N=62, n(\%)$ & $12(19)$ & $11(18)$ & $7(11)$ & $6(10)$ & $26(42)$ \\
\hline Non-U.S. ${ }^{a}$ & $6(50)$ & $7(64)$ & $5(71)$ & $2(33)$ & $16(62)$ \\
\hline U.S. & $6(50)$ & $4(36)$ & $2(29)$ & $4(67)$ & $10(38)$ \\
\hline Cancer & 1 (8) & - & - & - & - \\
\hline Cardiovascular & - & $3(27)$ & $3(43)$ & - & $5(19)$ \\
\hline Metabolic disorders & $2(17)$ & $1(9)$ & $1(14)$ & - & $6(23)$ \\
\hline Neurological & $1(8)$ & $4(36)$ & - & - & - \\
\hline Sleep disorders & - & $2(18)$ & - & - & $1(4)$ \\
\hline Substance abuse & - & - & - & - & $1(4)$ \\
\hline Weight management & $4(33)$ & - & - & $4(67)$ & $3(12)$ \\
\hline Other/Multiple & - & - & $1(14)$ & - & $1(4)$ \\
\hline
\end{tabular}

Age category, $n(\%)$

\begin{tabular}{|c|c|c|c|c|c|}
\hline$<20$ years old & $1(8)$ & $1(9)$ & - & - & $1(4)$ \\
\hline 21-39 years old & $1(8)$ & $2(18)$ & - & - & - \\
\hline 40-64 years old & $3(25)$ & $2(18)$ & $2(29)$ & $1(17)$ & $3(12)$ \\
\hline$\geq 65$ years old & $1(8)$ & $1(9)$ & - & - & $4(15)$ \\
\hline$>20$ years old ${ }^{c}$ & $6(50)$ & $4(36)$ & $5(71)$ & $5(83)$ & $18(69)$ \\
\hline Not reported & & $1(9)$ & & & \\
\hline
\end{tabular}

Feedback loop, $n(\%)$

\begin{tabular}{|c|c|c|c|c|c|}
\hline Counselors & 1 (8) & - & - & $1(17)$ & - \\
\hline Nurse & 1 (8) & - & $2(29)$ & - & 7 (27) \\
\hline Pharmacist & - & - & - & - & $1(4)$ \\
\hline Physician & $3(25)$ & $3(27)$ & $4(57)$ & 2 (33) & $7(27)$ \\
\hline Researchers & 1 (8) & $4(36)$ & - & - & - \\
\hline Self/Patient & $4(33)$ & - & - & 2 (33) & $5(19)$ \\
\hline Not reported & - & $3(27)$ & - & - & $1(4)$ \\
\hline Other ${ }^{d}$ & $2(17)$ & $1(9)$ & $1(14)$ & $1(17)$ & $5(19)$ \\
\hline
\end{tabular}

Results, $n(\%)$

\begin{tabular}{l|c|c|c|c|c}
\hline Negative & - & $1(9)$ & - & - & - \\
\hline Neutral & $5(42)$ & $5(45)$ & $1(14)$ & - & $5(19)$ \\
\hline Positive & $7(58)$ & $5(45)$ & $6(86)$ & $6(100)$ & $21(81)$ \\
\hline
\end{tabular}

${ }^{a}$ Non-U.S. countries included Canada, China, Taiwan, Korea, and several European countries (France, Italy, Spain, Denmark, Netherlands, Germany, Switzerland, and the United Kingdom).

"Disease category: "Other/multiple" includes patients with leg ulcers, glaucoma, systemic sclerosis, and rheumatoid arthritis.

'Age category: "Adults (>20 years)" refers to studies with more than one adult age category included (e.g., 35-70 years old).

${ }^{\mathrm{d}}$ Feedback loop: "Other" refers to multiple categories of feedback loop categories (i.e., physician and researcher), other healthcare professionals not listed, caregivers, or studies which did not explicitly report the end-user of the data. 


\section{VEGESNA ET AL.}

peer-reviewed literature. ${ }^{83,84}$ Large-scale implementation is challenging in terms of budget (cost of technology and infrastructure to monitor), educating patients in the use of devices, training providers in the collection and interpretation of results, as well as incorporation of the remote patient data into routine clinical practice. ${ }^{6,84,85}$ To our knowledge, this study is the first systematic review of RPM using noninvasive digital technologies examining any disease or condition spanning the last decade in the peer-reviewed literature. Similar to systematic reviews of other clinical interventions, we purposely applied the same level of methodological rigor, including quality appraisal, to identify the best available evidence to describe the key trends, patient and clinical characteristics, and outcomes measured by these technologies.

Several studies identified gaps that may be addressed by future research, including patient activation and costs. In terms of patient activation, new technologies such as smartphones/PDAs not only reduce resources in terms of patient visits but they may also promote health-related behavioral changes in patients by delivering convenient and individually tailored interventions. ${ }^{5-8,21,86}$ Furthermore, wireless transmission of patient data directly from devices to a centralized system may be the most cutting-edge technology available. ${ }^{5}$ These devices (typically biosensors or wearables) reduce patient burden and human error associated with manual entry and data transmission from the peripheral devices to the centralized data system.

In this systematic review, the few studies that reported cost outcomes found many costs associated with utilizing an RPM technology to be neutral or cost-saving compared with the control group. ${ }^{29,43,60,66,69,81}$ The majority of patients in studies reporting cost were $>50$ years of age, which may be due to the value of RPM data being highest among seniors due to the demand on provider's time. ${ }^{15,87}$ The growing interest in RPM for programs such as Medicare is expected to increase as the senior U.S. population grows and future studies may begin to explore cost-savings associated with these technologies. ${ }^{88}$ However, a recent RCT study found little evidence of differences in shortterm healthcare utilization and costs between patients with chronic conditions monitored through mobile health or digital medicine technology versus standard disease management. ${ }^{89}$ To better understand the appropriate use of RPM interventions using noninvasive digital technologies, future research should explore long-term healthcare utilization and costs in various patient populations such as those with chronic diseases.

\section{Limitations}

There are a few limitations that should be noted for our systematic review. The search string was very specific and may not have captured all articles. Although the majority of studies were RCT designs, observational cross-sectional studies were included, but no quality appraisal was performed. These studies are unlikely to impact the overall findings due to the descriptive nature of the results. In addition, the majority of studies were exploratory or pilot designs, with a high degree of variability in the objectives, populations, and outcomes reported; therefore, the information extracted was limited to broad categorization, subject to prespecified definitions, to assess commonality and trends. Many of the studies included in the systematic review enrolled relatively small patient populations; future clinical trials should assess the sample size required to have an adequately powered design and allow for a more generalizable population to assess the effectiveness of RPM technology on the outcomes of interest. In addition, the majority of the included studies reported short trial durations; however, to accurately assess the effectiveness of these technologies, the duration should align with the study objectives and clinical outcomes assessed (e.g., readmission rates). Discretion was used with the "feedback loop/end user" variable as this was not clearly described in many of the articles; however, we still felt it was an important aspect of these interventions and highly relevant to their overall value in healthcare. Many of the studies included only descriptive results; lack of robust measurement on the benefit of RPM on patient outcomes and care delivery was demonstrated. In terms of implementation, most studies did not report whether the data was ultimately added to the health system records, as previously stated this may be due to the exploratory nature of these studies.

\section{Conclusions}

Despite its potential, the rapid development of technological capabilities in the healthcare field has outpaced the capacity to implement many novel RPM interventions into real-world practice. Hence, scant evidence demonstrating improved health outcomes with noninvasive RPM interventions is available and even fewer studies have demonstrated any cost benefit. Based on this systematic review, there is a key trend toward using multicomponent interventions for the monitoring of chronic conditions in older populations. Further research utilizing robust study designs is needed to assess the efficacy and value of RPM technology for decision makers, developers, researchers, clinicians, and investors. This study will help decision makers better understand the current state of evidence available in peer-reviewed literature and assist in the planning of future studies that could address the existing gaps identified in this systematic review. Large-scale implementation is required to confirm the benefits 


\section{RPM VIA NONINVASIVE DIGITAL TECHNOLOGIES: A SYSTEMATIC REVIEW}

of RPM across sectors and populations in the health systems and identify the "optimal" patient for maximum utility of RPM. Barriers to implementation should also be assessed, including provider training, data reliability, security, and incorporation of RPM data into routine care.

\section{Acknowledgments}

Michelle A. Adams, BSJ, MA, of Write All, Inc., Sonoma, CA, Tara Cowling, MA, MSc, and Brittany Gerber, MA, of Medlior Health Outcomes Research Ltd. provided medical writing and editorial assistance for this article. Brooke Rakai, $\mathrm{PhD}$ for providing research assistance in screening, data extraction, and quality appraisal and Krista White, MA for quality appraisal. Rosemary Besrutschko, MLIS Manager, Novartis Knowledge Center Information Delivery, was the medical librarian who assisted in the EMBASE and OVID search.

\section{Disclosure Statement}

A.V. is a postdoctoral student from Jefferson College of Population Health and a US HEOR Fellow at Novartis Pharmaceuticals Corporation. M.T. is a postdoctoral student from Scott \& White Health Plan, University of Texas at Austin and a US HEOR Fellow at Novartis Pharmaceuticals Corporation. S.A. and M.A. are employees of Novartis Pharmaceuticals Corporation. Novartis Pharmaceuticals Corporation provided funding for this work.

\section{REFERENCES}

1. HRSA. What is Telehealth. Available at www.hrsa.gov/healthit/toolbox/ RuralHealthITtoolbox/Telehealth/whatistelehealth.html (last accessed December 4, 2015).

2. University of Miami. Telehealth definitions. Available at http:// telehealth.med.miami.edu/what-is-telehealth (last accessed October 15, 2015).

3. The National Alliance for Health Information Technology Report to the Office of the National Coordinator for Health Information Technology on Defining Key Health Information Technology Terms, released on April 28, 2008 LeadingAge.org/CAST.

4. LeadingAge CAST. Telehealth and Remote Patient Monitoring for LongTerm and PostAcute Care: A Primer and Provider Selection Guide 2015. Washington, DC: LeadingAge Center for Aging Services Technologies, 2015.

5. Steinhubl SR, Muse ED, Topol EJ. Can mobile health technologies transform health care? JAMA 2013:310:2395-2396

6. Steinhubl SR, Muse ED, Topol EJ. The emerging field of mobile health. Sci Trans/ Med 2015;7:283rv283.

7. Free $C$, Phillips $G$, Watson $L$, et al. The effectiveness of mobile-health technologies to improve health care service delivery processes: A systematic review and meta-analysis. PLOS Med 2013;10:e1001363.

8. Hamine $S$, Gerth-Guyette E, Faulx D, Green BB, Ginsburg AS. Impact of mHealth chronic disease management on treatment adherence and patient outcomes: A systematic review. J Med Internet Res 2015;17:e52.

9. Medicaid. Telemedicine definition. Available at www.medicaid.gov/ MedicaidCHIP-Program-Information/By-Topics/Delivery-Systems/ Telemedicine.html (last accessed December 4, 2015).
10. American Telemedicine Association. Telemedicine definition. Available at www.americantelemed.org/learn/what-is-telemedicine (last accessed December 4, 2015).

11. Field MJ, Grigsby J. Telemedicine and remote patient monitoring. JAMA 2002;288:423-425

12. Oh H, Rizo C, Enkin M, Alejandro J. What is eHealth (3): A systematic review of published definitions (3): A systematic review of published definitions. J Med Internet Res 2005;7:e1.

13. Sood S, Mbarika V, Jugoo $S$, et al. What is telemedicine? A collection of 104 peer-reviewed perspectives and theoretical underpinnings. Telemed J E Health 2007;13:573.

14. New England Healthcare Institute. Remote physiological monitoring: Research update 2009. Available at www.nehi.net/uploads/full_report/ rpm_research_update.pdf (last accessed December 4, 2015).

15. Darkins A, Ryan P, Kobb R, Foster L, Edmonson E, Wakefield B. Care Coordination/Home Telehealth: The systematic implementation of health informatics, home telehealth, and disease management to support the care of veteran patients with chronic conditions. Telemed J E Health 2008;14:1118.

16. Aslam S, Emmanuel P. Formulating a researchable question: A critical step for facilitating good clinical research. Indian J Sex Transm Dis 2010;31:47-50.

17. CASP. CASP checklist. 2014. Available at www.casp-uk.net/ (last accessed October 15, 2015).

18. Higgins JPT, Green S. Cochrane Handbook for Systematic Reviews of Interventions Version 5.1.0 [updated March 2011]. New York, NY: The Cochrane Collaboration, 2011

19. Moher D, Liberati A, Tetzlaff J, Altman DG; PRISMA Group. Preferred Reporting Items for Systematic Reviews and Meta-Analyses: The PRISMA statement. Int $J$ Surg 2010;8:336-341.

20. Allard M, Husky M, Catheline G, et al. Mobile technologies in the early detection of cognitive decline. PLoS One 2014;9:e112197.

21. Allen JK, Stephens J, Dennison Himmelfarb CR, Stewart KJ, Hauck S. Randomized controlled pilot study testing use of smartphone technology for obesity treatment. J Obes 2013;2013:151597.

22. DeVito Dabbs A, Dew MA, Myers B, et al. Evaluation of a hand-held, computerbased intervention to promote early self-care behaviors after lung transplant. Clin Transplant 2009;23:537-545.

23. Faurholt-Jepsen $M$, Ritz $C$, Frost $M$, et al. Mood instability in bipolar disorder type I versus type II-continuous daily electronic self-monitoring of illness activity using smartphones. J Affect Disord 2015;186:342-349.

24. Faurholt-Jepsen $M$, Vinberg $M$, Frost $M$, Christensen EM, Bardram JE, Kessing LV. Smartphone data as an electronic biomarker of illness activity in bipolar disorder. Bipolar Disord 2015;17:715-728.

25. Franc $S$, Borot $S$, Ronsin 0, et al. Telemedicine and type 1 diabetes: Is technology per se sufficient to improve glycaemic control? Diabetes Metab 2014;40:61-66

26. Fukuoka Y, Gay CL, Joiner KL, Vittinghoff E. A novel diabetes prevention intervention using a mobile App: A randomized controlled trial with overweight adults at risk. Am J Prev Med 2015;49:223-237.

27. Kearney N, McCann L, Norrie J, et al. Evaluation of a mobile phone-based, advanced symptom management system (ASyMS) in the management of chemotherapy-related toxicity. Support Care Cancer 2009;17:437-444

28. Nollen NL, Mayo MS, Carlson SE, Rapoff MA, Goggin KJ, Ellerbeck EF. Mobile technology for obesity prevention: A randomized pilot study in racial- and ethnic-minority girls. Am J Prev Med 2014;46:404-408.

29. Ryan D, Price D, Musgrave SD, et al. Clinical and cost effectiveness of mobile phone supported self monitoring of asthma: Multicentre randomised controlled trial. BMJ 2012;344:e1756.

30. Spring $B$, Duncan JM, Janke EA, et al. Integrating technology into standard weight loss treatment: A randomized controlled trial. JAMA Intern Med 2013;173:105-111 


\section{VEGESNA ET AL.}

31. Wang J, Sereika SM, Chasens ER, Ewing L, Matthews JT, Burke LE. Effect of adherence to self-monitoring of diet and physical activity on weight loss in a technologysupported behavioral intervention. Patient Prefer Adherence 2012;6:221-226.

32. Bombardini T, Gemignani V, Bianchini E, et al. Post-exercise contractility, diastolic function, and pressure: Operator-independent sensor-based intelligent monitoring for heart failure telemedicine. Cardiovasc Ultrasound 2009;7:21.

33. Dellaca R, Montserrat JM, Govoni L, Pedotti A, Navajas D, Farre R. Telemetric CPAP titration at home in patients with sleep apnea-hypopnea syndrome. Sleep Med 2011;12:153-157.

34. Dirienzo LN, Dirienzo LT, Baceski DA. Heart rate response to therapeutic riding in children with cerebral palsy: An exploratory study. Pediatr Phys Ther 2007;19:160-165.

35. Dorsch AK, Thomas $S, X u X$, Kaiser W, Dobkin BH; SIRRACT Investigators. SIRRACT: An international randomized clinical trial of activity feedback during inpatient stroke rehabilitation enabled by wireless sensing. Neurorehabil Neural Repair 2015;29:407-415.

36. Fox $N$, Hirsch-Allen AJ, Goodfellow $E$, et al. The impact of a telemedicine monitoring system on positive airway pressure adherence in patients with obstructive sleep apnea: A randomized controlled trial. Sleep 2012;35:477-481.

37. Lee YH, Hur SH, Sohn J, et al. Impact of home-based exercise training with wireless monitoring on patients with acute coronary syndrome undergoing percutaneous coronary intervention. J Korean Med Sci 2013;28:564-568.

38. Minassian A, Henry BL, Geyer MA, Paulus MP, Young JW, Perry W. The quantitative assessment of motor activity in mania and schizophrenia. $J$ Affect Disord 2010;120:200-206.

39. Palmas W, Pickering TG, Teresi J, et al. Telemedicine home blood pressure measurements and progression of albuminuria in elderly people with diabetes. Hypertension 2008;51:1282-1288.

40. Salarian A, Russmann H, Vingerhoets FJ, Burkhard PR, Aminian K. Ambulatory monitoring of physical activities in patients with Parkinson's disease. IEEE Trans Biomed Eng 2007;54:2296-2299.

41. Su R-HH, Hsu Y-L, Chan L, Lin H, Yang C-C. Assessing abnormal gaits of parkinson's disease patients using a wearable motion detector. Biomed Eng (Singapore) 2014;26:1-8.

42. Zheng YL, Yan BP, Zhang YT, Poon CC. An armband wearable device for overnight and cuff-less blood pressure measurement. IEEE Trans Biomed Eng 2014;61:2179-2186.

43. Dinesen $B$, Haesum LK, Soerensen N, et al. Using preventive home monitoring to reduce hospital admission rates and reduce costs: A case study of telehealth among chronic obstructive pulmonary disease patients. J Telemed Telecare 2012;18:221-225.

44. Guihot $A$, Becquemin MH, Couderc $\sqcup$, et al. Telemetric monitoring of pulmonary function after allogeneic hematopoietic stem cell transplantation. Transplantation 2007;83:554-560.

45. Hoefman E, van Weert HC, Boer KR, Reitsma J, Koster RW, Bindels PJ. Optimal duration of event recording for diagnosis of arrhythmias in patients with palpitations and light-headedness in the general practice. Fam Pract 2007;24:11-13.

46. Olson JA, Fouts AM, Padanilam BJ, Prystowsky EN. Utility of mobile cardiac outpatient telemetry for the diagnosis of palpitations, presyncope, syncope, and the assessment of therapy efficacy. J Cardiovasc Electrophysiol 2007;18:473-477.

47. Piga $M$, Tradori I, Pani D, et al. Telemedicine applied to kinesiotherapy for hand dysfunction in patients with systemic sclerosis and rheumatoid arthritis: Recovery of movement and telemonitoring technology. J Rheumatol 2014;41:1324-1333.

48. Stone RA, Rao RH, Sevick MA, et al. Active care management supported by home telemonitoring in veterans with type 2 diabetes: The DiaTel randomized controlled trial. Diabetes Care 2010;33:478-484.

49. Woodend AK, Sherrard H, Fraser M, Stuewe L, Cheung T, Struthers C. Telehome monitoring in patients with cardiac disease who are at high risk of readmission. Heart Lung 2008;37:36-45.

50. Anton SD, LeBlanc E, Allen HR, Karabetian C, Sacks F, Bray G, Williamson DA. Use of a computerized tracking system to monitor and provide feedback on dietary goals for calorie-restricted diets: The POUNDS LOST study. J Diabetes Sci Technol 2012;6:1216-1225.

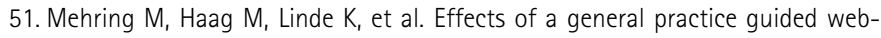
based weight reduction program-results of a cluster-randomized controlled trial. BMC Fam Pract 2013;14:76.

52. Rasmussen LM, Phanareth $\mathrm{K}$, Nolte $\mathrm{H}$, Backer $\mathrm{V}$. Internet-based monitoring of asthma: A long-term, randomized clinical study of 300 asthmatic subjects. J Allergy Clin Immunol 2005;115:1137-1142.

53. Thomas JG, Leahey TM, Wing RR. An automated internet behavioral weight-loss program by physician referral: A randomized controlled trial. Diabetes Care 2015;38:9-15.

54. Webber $\mathrm{KH}$, Rose SA. A pilot Internet-based behavioral weight loss intervention with or without commercially available portion-controlled foods. Obesity (Silver Spring) 2013;21:E354-E359.

55. Rogers K, Banis M, Falkenstein MJ, et al. Stepped care in the treatment of trichotillomania. J Consult Clin Psychol 2014;82:361-367.

56. Barnett NP, Tidey J, Murphy JG, Swift R, Colby SM. Contingency management for alcohol use reduction: A pilot study using a transdermal alcohol sensor. Drug Alcohol Depend 2011;118:391-399.

57. Carr $\sqcup$, Karvinen $K$, Peavler M, Smith R, Cangelosi K. Multicomponent intervention to reduce daily sedentary time: $A$ randomised controlled trial. $B M J$ Open 2013;3:e003261.

58. Chau JP, Lee DT, Yu DS, et al. A feasibility study to investigate the acceptability and potential effectiveness of a telecare service for older people with chronic obstructive pulmonary disease. Int J Med Inform 2012;81:674-682.

59. Dansky KH, Vasey J, Bowles K. Impact of telehealth on clinical outcomes in patients with heart failure. Clin Nurs Res 2008;17:182-199.

60. De San Miguel K, Smith J, Lewin G. Telehealth remote monitoring for community-dwelling older adults with chronic obstructive pulmonary disease. Telemed J E Health 2013;19:652-657.

61. Green BB, Cook AJ, Ralston JD, et al. Effectiveness of home blood pressure monitoring, Web communication, and pharmacist care on hypertension control: A randomized controlled trial. JAMA 2008;299:2857-2867.

62. Greene J, Sacks R, Piniewski B, Kil D, Hahn JS. The impact of an online social network with wireless monitoring devices on physical activity and weight loss. J Prim Care Community Health 2013;4:189-194.

63. Jan RL, Wang JY, Huang MC, Tseng SM, Su HJ, Liu LF. An internet-based interactive telemonitoring system for improving childhood asthma outcomes in Taiwan. Telemed J E Health 2007;13:257-268.

64. Jehn M, Donaldson $G$, Kiran $B$, et al. Tele-monitoring reduces exacerbation of COPD in the context of climate change-a randomized controlled trial. Environ Health 2013;12:99.

65. Jurgens C, Grossjohann R, Tost F. Distribution of mean, systolic and diastolic ocular perfusion pressure in telemedical homemonitoring of glaucoma patients. Ophthalmic Res 2012;48:208-211.

66. Koff PB, Jones RH, Cashman JM, Voelkel NF, Vandivier RW. Proactive integrated care improves quality of life in patients with COPD. Eur Respir J 2009;33:1031-1038.

67. Logan AG, Irvine MJ, Mclsaac WJ, et al. Effect of home blood pressure telemonitoring with self-care support on uncontrolled systolic hypertension in diabetics. Hypertension 2012;60:51-57.

68. Logan AG, Mclsaac WJ, Tisler A, et al. Mobile phone-based remote patient monitoring system for management of hypertension in diabetic patients. Am J Hypertens 2007;20:942-948.

69. Luley C, Blaik A, Gotz A, et al. Weight loss by telemonitoring of nutrition and physical activity in patients with metabolic syndrome for 1 year. J Am Coll Nutr 2014;33:363-374.

70. Mendelson M, Vivodtzev I, Tamisier $\mathrm{R}$, et al. CPAP treatment supported by telemedicine does not improve blood pressure in high cardiovascular risk OSA patients: A randomized, controlled trial. Sleep 2014;37:1863-1870. 


\section{RPM VIA NONINVASIVE DIGITAL TECHNOLOGIES: A SYSTEMATIC REVIEW}

71. Pedone $C$, Chiurco D, Scarlata S, Incalzi RA. Efficacy of multiparametric telemonitoring on respiratory outcomes in elderly people with COPD: A randomized controlled trial. BMC Health Serv Res 2013;13:82.

72. Pedone C, Rossi FF, Cecere A, Costanzo L, Antonelli Incalzi R. Efficacy of a physician-led multiparametric telemonitoring system in very old adults with heart failure. J Am Geriatr Soc 2015;63:1175-1180.

73. Shane-McWhorter $L$, Lenert $L$, Petersen $M$, et al. The Utah Remote Monitoring Project: Improving health care one patient at a time. Diabetes Technol Ther 2014; 16:653-660.

74. Shany $T$, Hession M, Pryce $D$, et al. Home telecare study for patients with chronic lung disease in the Sydney West Area Health Service. Stud Health Technol Inform 2010;161:139-148.

75. Tabak M, Vollenbroek-Hutten MM, van der Valk PD, van der Palen J, Tonis TM, Hermens HJ. Telemonitoring of daily activity and symptom behavior in patients with COPD. Int J Telemed App/ 2012;2012:438736.

76. van der Meer $V_{1}$ Bakker MJ, van den Hout WB, et al. Internet-based selfmanagement plus education compared with usual care in asthma: $A$ randomized trial. Ann Intern Med 2009;151:110-120.

77. Wakefield BJ, Holman JE, Ray A, et al. Effectiveness of home telehealth in comorbid diabetes and hypertension: A randomized, controlled trial. Telemed J E Health $2011 ; 17: 254-261$.

78. Wakefield BJ, Holman JE, Ray A, et al. Outcomes of a home telehealth intervention for patients with diabetes and hypertension. Telemed J E Health 2012;18:575-579.

79. Widmer RJ, Allison TG, Lerman LO, Lerman A. Digital health intervention as an adjunct to cardiac rehabilitation reduces cardiovascular risk factors and rehospitalizations. J Cardiovasc Trans/ Res 2015;8:283-292.

80. Wijsman CA, Westendorp RG, Verhagen EA, et al. Effects of a web-based intervention on physical activity and metabolism in older adults: Randomized controlled trial. J Med Internet Res 2013;15:e233.

81. Scalvini S, Zanelli E, Martinelli G, Baratti D, Giordano A, Glisenti F. Cardiac event recording yields more diagnoses than 24-hour Holter monitoring in patients with palpitations. J Telemed Telecare 2005;11(Suppl 1):14-16.

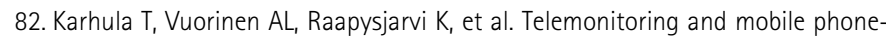
based health coaching among finnish diabetic and heart disease patients: Randomized controlled trial. J Med Internet Res 2015;17:e153.
83. Nakamura $N$, Koga $T$, Iseki $H$. A meta-analysis of remote patient monitoring for chronic heart failure patients. J Telemed Telecare 2014;20:11-17.

84. Davis MM, Freeman M, Kaye J, Vuckovic N, Buckley DI. A systematic review of clinician and staff views on the acceptability of incorporating remote monitoring technology into primary care. Telemed J E Health 2014;20:428-438.

85. Li J, Wilson LS. Telehealth trends and the challenge for infrastructure. Telemed J E Health 2013;19:772-779.

86. Free C, Phillips G, Galli L, et al. The effectiveness of mobile-health technologybased health behaviour change or disease management interventions for health care consumers: A systematic review. PLoS Med 2013;10:e1001362.

87. Wireless Sensor Technology: Aging Services Applications and Associated Risks. Available at www.cna.com/vcm_content/CNA/internet/ Static\%20File\%20for\%20Download/Risk\%20Control/Medical\%20Services/ WirelessSensorTechnologyAgingServicesApplicationsandAssociatedRisks.pdf (last accessed December 4, 2015).

88. Neufeld JD, Doarn CR. Telemedicine spending by Medicare: A snapshot from 2012. Telemed J E Health 2015; 21:686-693.

89. Bloss CS, Wineinger NE, Peters $M$, et al. A prospective randomized trial examining health care utilization in individuals using multiple smartphoneenabled biosensors. PeerJ 2016;4:e1554.

Address correspondence to: Ashok Vegesna, PharmD Jefferson College of Population Health/ Novartis Pharmaceuticals One Health Plaza East Hanover, NJ 07936-1080

E-mail: ashok.vegesna@novartis.com

Received: February 23, 2016

Revised: March 18, 2016

Accepted: March 24, 2016 